\title{
Superheavy nuclei and giant quasi-atoms
}

\author{
Valery Zagrebaev ${ }^{\mathrm{a}}$ and Walter Greiner ${ }^{\mathrm{b}}$ \\ ${ }^{a}$ Flerov Laboratory of Nuclear Reactions, JINR, Dubna, Moscow Region, Russia \\ ${ }^{\text {b} F I A S, ~ J . W . ~ G o e t h e-U n i v e r s i t a ̈ t, ~ F r a n k f u r t, ~ G e r m a n y ~}$
}

Dynamics of heavy-ion low energy collisions is studied within the realistic model based on multi-dimensional Langevin equations. Symmetric and asymmetric fusion reactions leading to formation of superheavy nuclei are analyzed. Collisions of transactinide nuclei are investigated as an alternative way for production of neutron-rich superheavy elements. In many events lifetime of the composite giant system consisting of two touching nuclei turns out to be rather long $\left(\geq 10^{-20} \mathrm{~s}\right)$; sufficient for observing line structure in spontaneous positron emission from super-strong electric fields, a fundamental QED process.

\section{INTRODUCTION}

The mechanism of strongly damped collisions between very heavy nuclei was studied extensively about twenty years ago. Among others, there had been great interest in the use of heavy-ion transfer reactions with actinide targets to produce new nuclear species in the transactinide region. The cross sections were found to decrease rapidly with increasing atomic number of surviving target-like fragments. However, Fm and $\mathrm{Md}$ neutron-rich isotopes have been produced at the level of $0.1 \mu b[1,2]$. It was observed also that nuclear structure may strongly influence nucleon flow in dissipative collisions with actinide targets.

Renewed interest to collisions of transactinide nuclei is conditioned by the necessity to clarify much better than before the dynamics of heavy nuclear systems at low excitation energies and by a search for new ways for production of neutron rich superheavy (SH) nuclei and isotopes. SH elements obtained in "cold" fusion reactions with $\mathrm{Pb}$ or Bi target are situated along the proton drip line being very neutron-deficient with a short half-life. In fusion of actinides with ${ }^{48} \mathrm{Ca}$ more neutron-rich $\mathrm{SH}$ nuclei are produced with much longer half-life. But they are still far from the center of the predicted "island of stability" formed by the neutron shell $\mathrm{N}=184$. In the "cold" fusion, the cross sections for formation of SH nuclei decrease very fast with increasing charge of the projectile and become less than $1 \mathrm{pb}$ for $\mathrm{Z} \geq 112$. On the other hand, the heaviest transactinide, Cf, which can be used as a target in the second method, being fused with ${ }^{48} \mathrm{Ca}$ leads to the $\mathrm{SH}$ nucleus with $\mathrm{Z}=118$. Using the next nearest elements instead of ${ }^{48} \mathrm{Ca}$ (e.g., ${ }^{50} \mathrm{Ti},{ }^{54} \mathrm{Cr}$, etc.) in fusion reactions with actinides is expected less encouraging, though experiments of such kind are planned to be performed. In this connection other ways to the production of SH elements in the region of the "island of stability" should be searched for.

Recently a new model has been proposed [3] for simultaneous description of all these 
strongly coupled processes: deep inelastic (DI) scattering, quasi-fission (QF), fusion, and regular fission. In this paper we apply this model for analysis of low-energy dynamics of heavy nuclear systems formed in nucleus-nucleus collisions at the energies around the Coulomb barrier. Among others there is the purpose to find an influence of the shell structure of the driving potential (in particular, deep valley caused by the double shell closure $\mathrm{Z}=82$ and $\mathrm{N}=126$ ) on formation of compound nucleus $(\mathrm{CN})$ in mass asymmetric collisions and on nucleon rearrangement between primary fragments in more symmetric collisions of actinide nuclei. In the first case, discharge of the system into the lead valley (normal or symmetrizing quasi-fission) is the main reaction channel, which decreases significantly the probability of $\mathrm{CN}$ formation. In collisions of heavy transactinide nuclei $(\mathrm{U}+\mathrm{Cm}$, etc. $)$, we expect that the existence of this valley may noticeably increase the yield of surviving neutron-rich superheavy nuclei complementary to the projectile-like fragments (PLF) around lead ("inverse" or anti-symmetrizing quasi-fission reaction mechanism). Direct time analysis of the collision process allows us to estimate also the lifetime of the composite system consisting of two touching heavy nuclei with total charge Z $>180$. Such "long-living" configurations (if they exist) may lead to spontaneous positron emission from super-strong electric fields of giant quasi-atoms by a static QED process (transition from neutral to charged QED vacuum) [4].

\section{ADIABATIC DYNAMICS OF HEAVY NUCLEAR SYSTEM}

At incident energies around the Coulomb barrier in the entrance channel the fusion probability is about $10^{-3}$ for mass asymmetric reactions induced by ${ }^{48} \mathrm{Ca}$ and much less for more symmetric combinations used in the "cold synthesis". DI scattering and QF are the main reaction channels here, whereas the fusion probability [formation $\mathrm{CN}$ ] is extremely small. To estimate such a small quantity for CN formation probability, first of all, one needs to be able to describe well the main reaction channels, namely DI and QF. Moreover, the quasi-fission processes are very often indistinguishable from the DI scattering and from regular fission, which is the main decay channel of excited heavy CN.

To describe properly and simultaneously the strongly coupled DI, QF and fusion-fission processes of low-energy heavy-ion collisions we have to choose, first, the unified set of degrees of freedom playing the principal role both at approaching stage and at the stage of separation of reaction fragments. Second, we have to determine the unified potential energy surface (depending on all the degrees of freedom) which regulates all the processes. Finally, the corresponding equations of motion should be formulated to perform numerical analysis of the studied reactions. In contrast with other models, we take into consideration all the degrees of freedom necessary for description of all the reaction stages. Thus, we need not to split artificially the whole reaction into several stages. Moreover, in that case unambiguously defined initial conditions are easily formulated at large distance, where only the Coulomb interaction and zero-vibrations of the nuclei determine the motion. The distance between the nuclear centers $R$ (corresponding to the elongation of a mono-nucleus), dynamic spheroidal-type surface deformations $\beta_{1}$ and $\beta_{2}$, mutual in-plane orientations of deformed nuclei $\varphi_{1}$ and $\varphi_{2}$, and mass asymmetry $\eta=\frac{A_{1}-A_{2}}{A_{1}+A_{2}}$ are probably the relevant degrees of freedom in fusion-fission dynamics.

The two-center shell model [5] seems to be most appropriate for calculation of the 
adiabatic potential energy surface. However, the simplest version of this model, using standard parametrization of the macroscopic (liquid drop) part of the total energy, does not reproduce correctly values of the nucleus-nucleus interaction potential for well separated nuclei and at contact point. The same holds for the value of the Coulomb barrier and the depth of potential pocket at contact. To overcome these problems we used the adiabatic potential energy of the nuclear system calculated within the semi-empirical twocore model of nucleon collectivization [6] based on the two-center shell model idea. The two-core model gives an explicit (experimental) value of the nucleus-nucleus interaction energy for well separated nuclei and quite realistic heights of the Coulomb barriers, that is very important for the description of near-barrier heavy ion reactions.

A choice of dynamic equations for the considered degrees of freedom is also not so evident. The main problem here is a proper description of nucleon transfer and change of the mass asymmetry which is a discrete variable by its nature. The corresponding inertia parameter $\mu_{\eta}$, being calculated within the Werner-Wheeler approach, becomes infinite at contact (scission) point and for separated nuclei. In Ref. [3] the inertialess Langevin type equation for the mass asymmetry has been derived from the corresponding master equation for the distribution function. Finally we have a set of 13 coupled Langevin type equations for 7 degrees of freedom [3] which are solved numerically.

The cross sections for all the processes are calculated in a simple and natural way. A large number of events (trajectories) are tested for a given impact parameter. Those events, in which the nuclear system overcame the fission barrier from the outside and entered the region of small deformations and elongations, are treated as fusion (CN formation). The other events correspond to quasi-elastic, DI and QF processes. Subsequent decay of the excited $\mathrm{CN}(C \rightarrow B+x n+N \gamma)$ is described then within the statistical model using an explicit expression for survival probability, which directly takes into account the Maxwell-Boltzmann energy distribution of evaporated neutrons [7]. The double differential cross-sections are calculated as follows

$\frac{d^{2} \sigma_{\eta}}{d \Omega d E}(E, \theta)=\int_{0}^{\infty} b d b \frac{\Delta N_{\eta}(b, E, \theta)}{N_{\text {tot }}(b)} \frac{1}{\sin (\theta) \Delta \theta \Delta E}$.

Here $\Delta N_{\eta}(b, E, \theta)$ is the number of events at a given impact parameter $b$ in which the system enters into the channel $\eta$ (definite mass asymmetry value) with kinetic energy in the region $(E, E+\Delta E)$ and center-of-mass outgoing angle in the region $(\theta, \theta+\Delta \theta), N_{\text {tot }}(b)$ is the total number of simulated events for a given value of impact parameter. In collisions of deformed nuclei an averaging over initial orientations is performed. Expression (1) describes the mass, energy and angular distributions of the primary fragments formed in the binary reaction (both in DI and in QF processes). Subsequent de-excitation cascades of these fragments via emission of light particles and gamma-rays in competition with fission were taken into account explicitly for each event within the statistical model leading to the final mass and energy distributions of the reaction fragments. The model allows us to perform also a time analysis of the studied reactions. Each tested event is characterized by the reaction time $\tau_{\text {int }}$, which is calculated as a difference between re-separation (scission) and contact times. 


\section{DEEP INELASTIC SCATTERING OF HEAVY NUCLEI}

At first we applied the model to describe available experimental data on low-energy damped collision of very heavy nuclei, ${ }^{136} \mathrm{Xe}+{ }^{209} \mathrm{Bi}[8]$, where the DI process should dominate due to expected prevalence of the Coulomb repulsion over nuclear attraction. The adiabatic potential energy surface of this nuclear system is shown on the left panel of Fig. 1 in the space of elongation and mass asymmetry at zero dynamic deformations. The colliding nuclei are very compact with almost closed shells and the potential energy has only one deep valley (just in the entrance channel, $\eta \sim 0.21$ ) giving rather simple mass distribution of the reaction fragments. In that case the reaction mechanism depend mainly on the nucleus-nucleus potential at contact distance, on the friction forces at this region (which determine the energy loss) and on nucleon transfer rate at contact. Note, that there is a well pronounced plateau at contact configuration in the region of zero mass asymmetry (see Fig. 1, right panel). It becomes even lower with increasing the deformations and corresponds to formation of the nuclear system consisting of strongly deformed touching fragments ${ }^{172} \mathrm{Er}+{ }^{173} \mathrm{Tm}$ (see Fig. 1), which means that a significant mass rearrangement may occur here leading to additional time delay of the reaction.
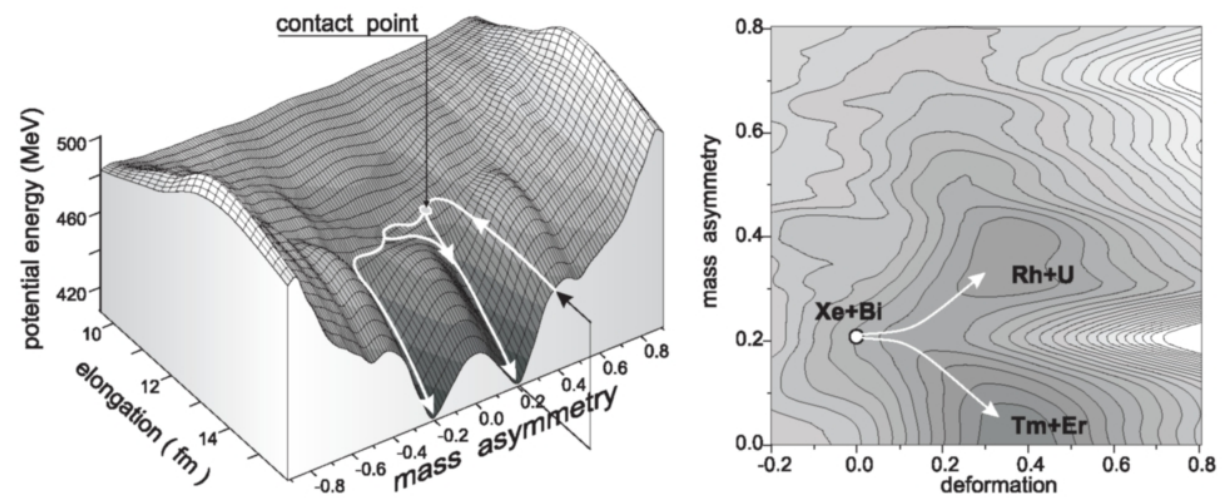

Figure 1. Driving potential for the nuclear system formed in ${ }^{136} \mathrm{Xe}+{ }^{209} \mathrm{Bi}$ collision at fixed deformations (left) and at contact configuration (right). The solid lines with arrows show schematically (without fluctuations) most probable trajectories.

On the right panel of Fig. 1 the landscape of the potential energy is shown at contact configuration depending on mass asymmetry and deformation of the fragments. As can be seen, after contact and before re-separation the nuclei aim to become more deformed. Moreover, beside a regular diffusion (caused by the fluctuations), the final mass distribution is determined also by the two well marked driving paths leading the system to more and to less symmetric configurations. They are not identical and this leads to the asymmetric mass distribution of the primary fragments, see Fig. 2(c).

In Fig. 2 the angular, energy and charge distributions of the Xe-like fragments are shown comparing with our calculations (histograms). In accordance with experimental conditions only the events with the total kinetic energy in the region of $260 \leq E \leq 546 \mathrm{MeV}$ and with the scattering angles in the region of $40^{\circ} \leq \theta_{\text {c.m. }} \leq 100^{\circ}$ were accumulated. 
The total cross section corresponding to all these events is about $2200 \mathrm{mb}$ (experimental estimation is $2100 \mathrm{mb}[8]$ ). Due to the rather high excitation energy sequential fission of the primary heavy fragments may occur in this reaction (mainly those heavier than $\mathrm{Bi}$ ). In the experiment the yield of the heavy fragments was found to be about $30 \%$ less comparing with Xe-like fragments. Our calculation gives $354 \mathrm{mb}$ for the cross section of sequential-fission, which is quite comparable with experimental data. Mass distribution of the fission fragments is shown in Fig. 2(c) by the dotted histogram. Note that it is a contamination with sequential fission products of heavy primary fragments leading to the bump around $\mathrm{Z}=40$ in the experimental charge distribution in Fig. 2(c).
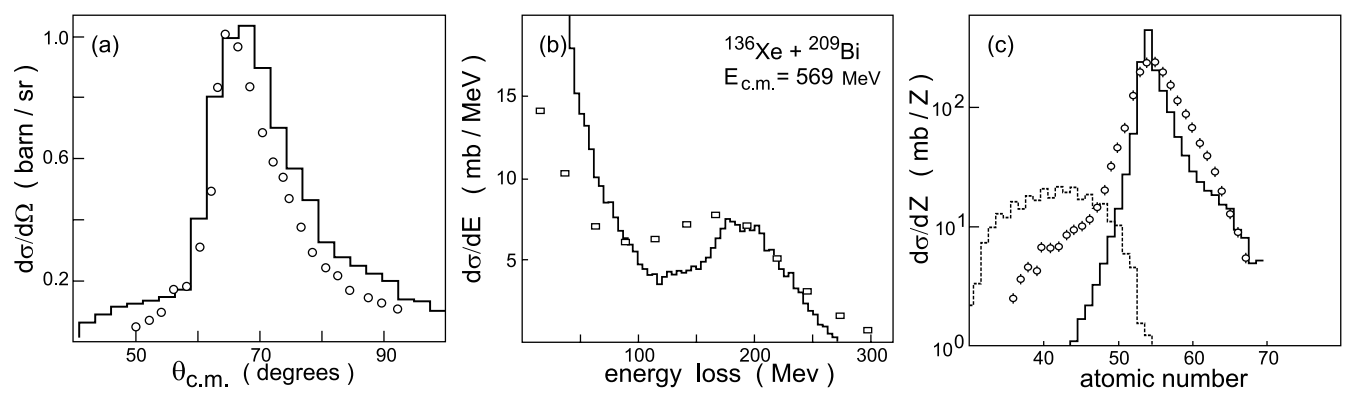

Figure 2. Angular (a), energy-loss (b) and charge (c) distributions of the Xe-like fragments obtained in the ${ }^{136} \mathrm{Xe}+{ }^{209} \mathrm{Bi}$ reaction at $E_{\text {c.m. }}=568 \mathrm{MeV}$. Experimental data are taken from Ref. [8]. For other notations see the text.

\section{SYNTHESIS OF SUPERHEAVY NUCLEI}

Analysis of the near-barrier ${ }^{48} \mathrm{Ca}+{ }^{248} \mathrm{Cm}$ collisions (leading to formation of a superheavy nucleus), in which the QF process plays a dominant role, also demonstrated quite well agreement of our calculations with the experimental mass and energy distributions of DI and QF reaction products as well as with the cross section for CN formation [9]. Within our approach we estimated also a possibility of SH element production in fusion reactions of symmetric nuclei (fission-like fragments). In such reactions uncertainty in calculation of very small cross section for $\mathrm{CN}$ formation is rather large. Dashed and solid curves in Fig. 3(a) reflect this uncertainty in our estimations of the evaporation residue (EvR) cross sections in ${ }^{136} \mathrm{Xe}+{ }^{136} \mathrm{Xe}$ fusion reactions. If the experiment (planned to be performed in Dubna) will give the EvR cross sections at the level of few picobarns for this reaction then we may really dream about using neutron-rich accelerated fission fragments for production of SH elements in the region of the "island of stability" (e.g., ${ }^{132} \mathrm{Sn}+{ }^{176} \mathrm{Yb} \rightarrow{ }^{308} 120$ ).

Another possibility for synthesis of SH elements heavier than 118 are the asymmetric fusion reactions of nuclei heavier than ${ }^{48} \mathrm{Ca}$ with transuranium targets. Estimated EvR cross sections for production of element 120 in the ${ }^{54} \mathrm{Cr}+{ }^{248} \mathrm{Cm}$ fusion reaction are shown in Fig. 3(b) as compared with the ${ }^{48} \mathrm{Ca}+{ }^{248} \mathrm{Cm}$ reaction. For both cases we used the fission barriers of CN predicted by the macro-microscopical model [10], which gives much lower fission barrier for ${ }^{302} 120$ nucleus in comparison with ${ }^{296} 116$. However, full microscopic models based on the self-consistent Hartree-Fock calculations [11] predict much higher 
fission barriers for the nucleus ${ }^{302} 120$ (up to $10 \mathrm{MeV}$ for the Skyrme forces). This means that the $3 \mathrm{n}$ and $4 \mathrm{n}$ EvR cross sections in the ${ }^{54} \mathrm{Cr}+{ }^{248} \mathrm{Cm}$ fusion reactions could be three orders of magnitudes higher as compared with those shown in Fig. 3(b).
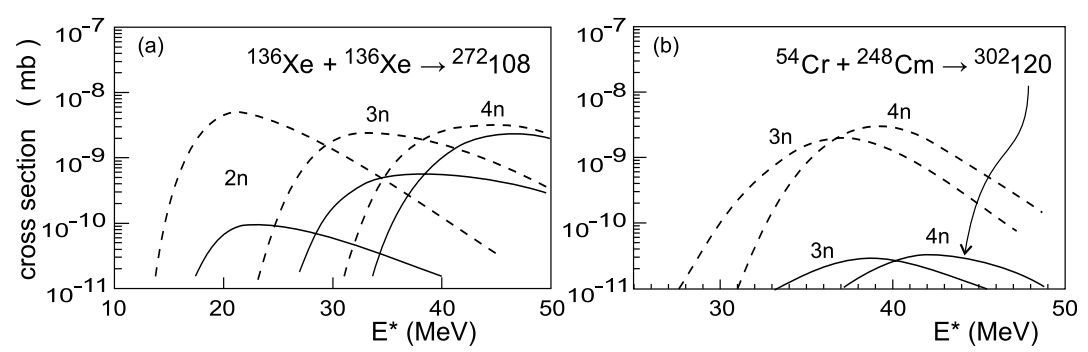

Figure 3. Evaporation residue cross sections in ${ }^{136} \mathrm{Xe}+{ }^{136} \mathrm{Xe}$ (a) and ${ }^{54} \mathrm{Cr}+{ }^{248} \mathrm{Cm}$ (b) fusion reactions (see the text). Dashed curves in (b) correspond to the case of ${ }^{48} \mathrm{Ca}+{ }^{248} \mathrm{Cm}$.

\section{LOW-ENERGY COLLISIONS OF TRANSACTINIDE NUCLEI}

Reasonable agreement of our calculations with experimental data on low-energy DI and QF reactions induced by heavy ions stimulated us to study the reaction dynamics of very heavy transactinide nuclei. The purpose was to find an influence of the shell structure of the driving potential (in particular, deep valley caused by the double shell closure $\mathrm{Z}=82$ and $\mathrm{N}=126$ ) on nucleon rearrangement between primary fragments. In Fig. 4 the potential energies are shown depending on mass rearrangement at contact configuration of the nuclear systems formed in ${ }^{48} \mathrm{Ca}+{ }^{248} \mathrm{Cm}$ and ${ }^{232} \mathrm{Th}+{ }^{250} \mathrm{Cf}$ collisions. The lead valley evidently reveals itself in both cases (for ${ }^{48} \mathrm{Ca}+{ }^{248} \mathrm{Cm}$ system there is also a tin valley). In the first case $\left({ }^{48} \mathrm{Ca}+{ }^{248} \mathrm{Cm}\right)$, discharge of the system into the lead valley (normal or symmetrizing quasi-fission) is the main reaction channel, which decreases significantly the probability of $\mathrm{CN}$ formation. In collisions of heavy nuclei ( $\mathrm{Th}+\mathrm{Cf}, \mathrm{U}+\mathrm{Cm}$ and so on) we expect that the existence of this valley may noticeably increase the yield of surviving neutron-rich superheavy nuclei complementary to the projectile-like fragments around ${ }^{208} \mathrm{~Pb}$ ("inverse" or anti-symmetrizing quasi-fission process).
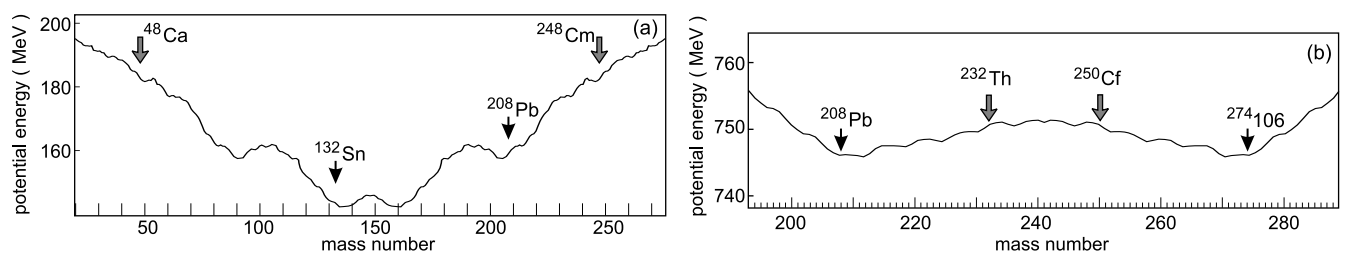

Figure 4. Potential energy at contact "nose-to-nose" configuration for the two nuclear systems formed in ${ }^{48} \mathrm{Ca}+{ }^{248} \mathrm{Cm}$ (a) and ${ }^{232} \mathrm{Th}+{ }^{250} \mathrm{Cf}$ (b) collisions.

Direct time analysis of the reaction dynamics allows us to estimate also the lifetime of the composite system consisting of two touching heavy nuclei with total charge $\mathrm{Z}>180$. 
Such "long-living" configurations may lead to spontaneous positron emission from superstrong electric field of giant quasi-atoms by a static QED process (transition from neutral to charged QED vacuum) [4]. About twenty years ago an extended search for this fundamental process was carried out and narrow line structures in the positron spectra were first reported at GSI. Unfortunately these results were not confirmed later, neither at ANL, nor in the last experiments performed at GSI. These negative finding, however, were contradicted by Jack Greenberg (private communication and supervised thesis at Wright Nuclear Structure Laboratory, Yale university). Thus the situation remains unclear, while the experimental efforts in this field have ended. We hope that new experiments and new analysis, performed according to the results of our dynamical model, may shed additional light on this problem and also answer the principal question: are there some reaction features (triggers) testifying a long reaction delays? If they are, new experiments should be planned to detect the spontaneous positrons in the specific reaction channels.
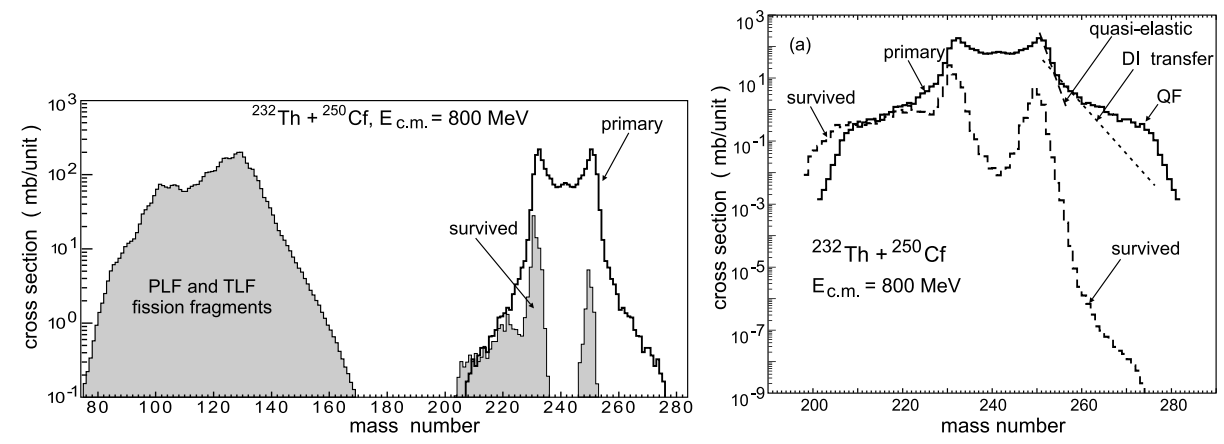

Figure 5. Mass distributions of primary (solid histogram), surviving and sequential fission fragments (hatched areas) in the ${ }^{232} \mathrm{Th}+{ }^{250} \mathrm{Cf}$ collision at $800 \mathrm{MeV}$ center-of-mass energy. On the right the result of longer calculation is shown.

Using the same parameters of nuclear viscosity and nucleon transfer rate as for the system $\mathrm{Xe}+\mathrm{Bi}$ we calculated the yield of primary and surviving fragments formed in the ${ }^{232} \mathrm{Th}+{ }^{250} \mathrm{Cf}$ collision at $800 \mathrm{MeV}$ center-of mass energy. Low fission barriers of the colliding nuclei and of most of the reaction products jointly with rather high excitation energies of them in the exit channel will lead to very low yield of surviving heavy fragments. Indeed, sequential fission of the projectile-like and target-like fragments dominate in these collisions, see Fig. 5. At first sight, there is no chances to get surviving superheavy nuclei in such reactions. However, as mentioned above, the yield of the primary fragments will increase due to the QF effect (lead valley) as compared to the gradual monotonic decrease typical for damped mass transfer reactions. Secondly, with increasing neutron number the fission barriers increase on average (also there is the closed sub-shell at $\mathrm{N}=162$ ). Thus we may expect a non-negligible yield (at the level of $1 \mathrm{pb}$ ) of surviving superheavy neutron rich nuclei produced in these reactions [12].

Result of much longer calculations is shown on the right panel of Fig. 5. The pronounced shoulder can be seen in the mass distribution of the primary fragments near the mass number $\mathrm{A}=208$ (274). It is explained by the existence of a valley in the potential energy 
surface [see Fig. 4(b)], which corresponds to the formation of doubly magic nucleus ${ }^{208} \mathrm{~Pb}$ $(\eta=0.137)$. The emerging of the nuclear system into this valley resembles the well-known quasi-fission process and may be called "inverse (or anti-symmetrizing) quasi-fission" (the final mass asymmetry is larger than the initial one). For $\eta>0.137$ (one fragment becomes lighter than lead) the potential energy sharply increases and the mass distribution of the primary fragments decreases rapidly at $\mathrm{A}<208(\mathrm{~A}>274)$.
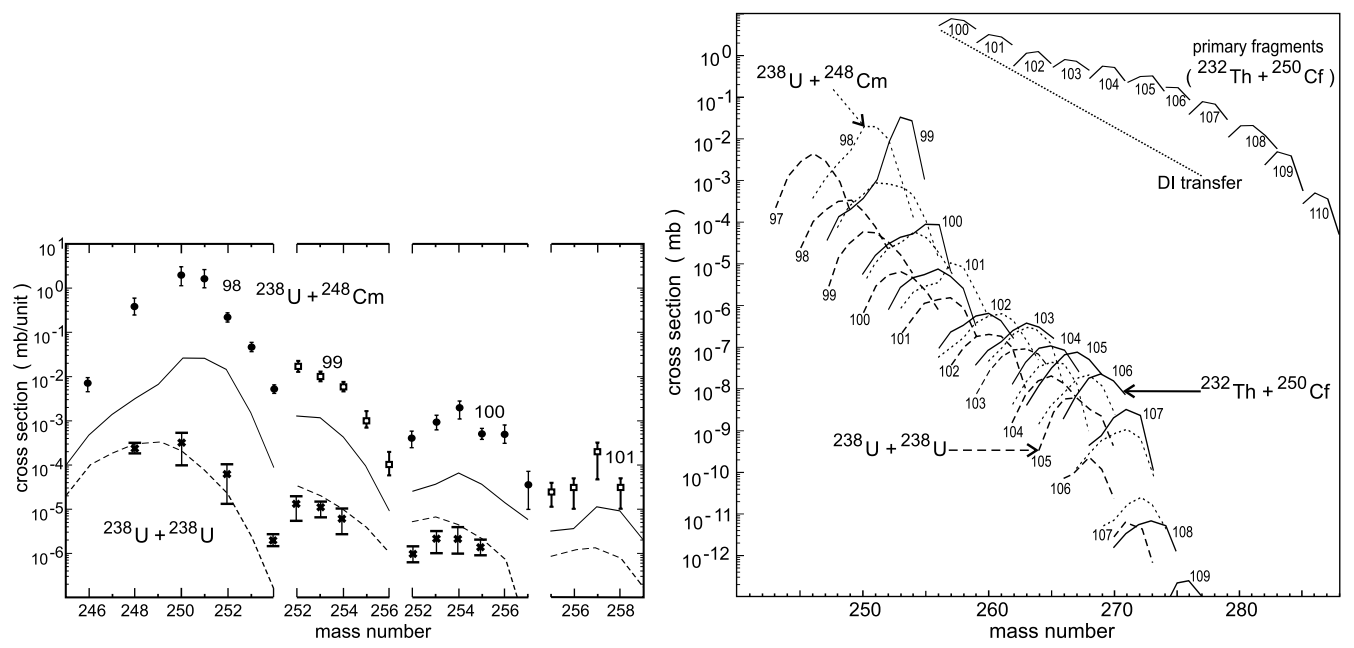

Figure 6. (Left panel) Experimental and calculated yields of the elements $98 \div 101$ in the reactions ${ }^{238} \mathrm{U}+{ }^{238} \mathrm{U}$ (crosses) [1] and ${ }^{238} \mathrm{U}+{ }^{248} \mathrm{Cm}$ (circles and squares) [2]. (Right panel) Predicted yields of superheavy nuclei in collisions of ${ }^{238} \mathrm{U}+{ }^{238} \mathrm{U}$ (dashed), ${ }^{238} \mathrm{U}+{ }^{248} \mathrm{Cm}$ (dotted) and ${ }^{232} \mathrm{Th}+{ }^{250} \mathrm{Cf}$ (solid lines) at $800 \mathrm{MeV}$ center-of-mass energy. Solid curves in upper part show isotopic distributions of primary fragments in the Th+Cf reaction.

In Fig. 6 the available experimental data on the yield of $\mathrm{SH}$ nuclei in collisions of ${ }^{238} \mathrm{U}+{ }^{238} \mathrm{U}$ [1] and ${ }^{238} \mathrm{U}+{ }^{248} \mathrm{Cm}[2]$ are compared with our calculations. The estimated isotopic yields of survived $\mathrm{SH}$ nuclei in the ${ }^{232} \mathrm{Th}+{ }^{250} \mathrm{Cf},{ }^{238} \mathrm{U}+{ }^{238} \mathrm{U}$ and ${ }^{238} \mathrm{U}+{ }^{248} \mathrm{Cm}$ collisions at $800 \mathrm{MeV}$ center-of-mass energy are shown on the right panel of Fig. 6. Thus, as we can see, there is a real chance for production of the long-lived neutron-rich SH nuclei in such reactions. As the first step, chemical identification and study of the nuclei up to ${ }_{107}^{274} \mathrm{Bh}$ produced in the reaction ${ }^{232} \mathrm{Th}+{ }^{250} \mathrm{Cf}$ may be performed.

The time analysis of the reactions studied shows that in spite of absence of an attractive potential pocket the system consisting of two very heavy nuclei may hold in contact rather long in some cases. During this time the giant nuclear system moves over the multidimensional potential energy surface with almost zero kinetic energy (result of large nuclear viscosity). The total reaction time distribution, $\frac{d \sigma}{d \log (\tau)}(\tau$ denotes the time after the contact of two nuclei), is shown in Fig. 7 for the ${ }^{238} \mathrm{U}+{ }^{248} \mathrm{Cm}$ collision. The dynamic deformations are mainly responsible here for the time delay of the nucleus-nucleus collision. Ignoring the dynamic deformations in the equations of motion significantly decreases the reaction time, see Fig. 7(a). With increase of the energy loss and mass 
transfer the reaction time becomes longer and its distribution becomes more narrow.

As mentioned earlier, the lifetime of a giant composite system more than $10^{-20} \mathrm{~s}$ is quite enough to expect positron line structure emerging on top of the dynamical positron spectrum due to spontaneous $e^{+} e^{-}$production from the supercritical electric fields as a fundamental QED process ("decay of the vacuum") [4]. The absolute cross section for long events is found to be maximal just at the beam energy ensuring the two nuclei to be in contact, see Fig. 7(c). The same energy is also optimal for the production of the most neutron-rich SH nuclei. Of course, there are some uncertainties in the used parameters, mostly in the value of nuclear viscosity. However we found only a linear dependence of the reaction time on the strength of nuclear viscosity, which means that the obtained reaction time distribution is rather reliable, see logarithmic scale on both axes in Fig. 7(a).
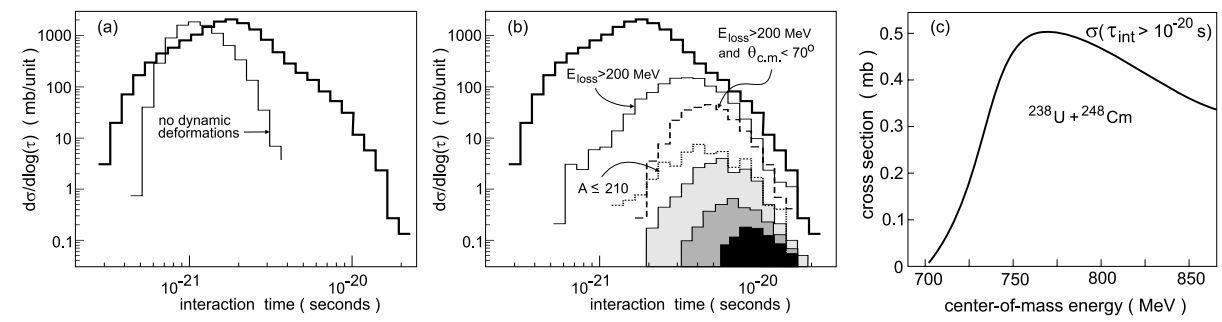

Figure 7. Reaction time distributions for the ${ }^{238} \mathrm{U}+{ }^{248} \mathrm{Cm}$ collision at $800 \mathrm{MeV}$ center-ofmass energy. Thick solid histograms correspond to all events with energy loss more than $30 \mathrm{MeV}$. (a) Thin solid histogram shows the effect of switching-off dynamic deformations. (b) Thin solid, dashed and dotted histograms show reaction time distributions in the channels with formation of primary fragments with $\mathrm{E}_{\text {loss }}>200 \mathrm{MeV}, \mathrm{E}_{\text {loss }}>200 \mathrm{MeV}$ and $\theta_{\text {c.m. }}<70^{\circ}$ and $\mathrm{A} \leq 210$, correspondingly. Hatched areas show time distributions of events with formation of the primary fragments with $\mathrm{A} \leq 220$ (light gray), $\mathrm{A} \leq 210$ (gray), $\mathrm{A} \leq 204$ (dark) having $\mathrm{E}_{\text {loss }}>200 \mathrm{MeV}$ and $\theta_{\text {c.m. }}<70^{\circ}$. (c) Cross section for events with interaction time longer than $10^{-20} \mathrm{~s}$.
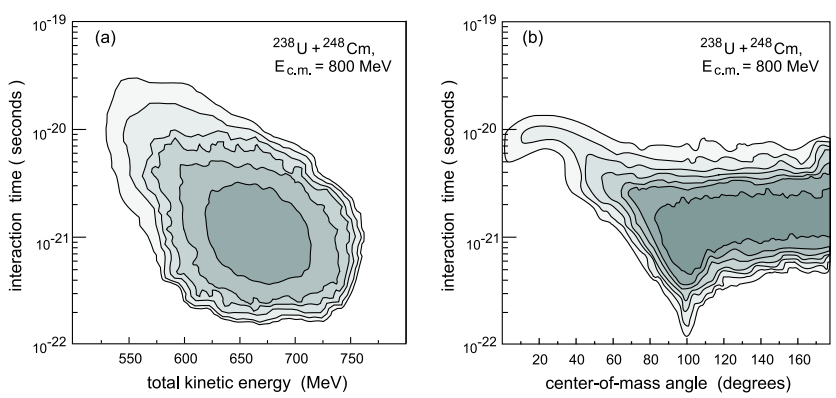

Figure 8. Energy-time (a) and angular-time (b) distributions of primary fragments in the ${ }^{238} \mathrm{U}+{ }^{248} \mathrm{Cm}$ collision at $800 \mathrm{MeV}\left(\mathrm{E}_{\text {loss }}>15 \mathrm{MeV}\right)$.

Formation of the background positrons in these reactions forces one to find some additional trigger for the longest events. Such long events correspond to the most damped collisions with formation of mostly excited primary fragments decaying by fission, see 
Figs. 8(a). However there is also a chance for production of the primary fragments in the region of doubly magic nucleus ${ }^{208} \mathrm{~Pb}$, which could survive against fission due to nucleon evaporation. The number of the longest events depends weakly on impact parameter up to some critical value. On the other hand, in the angular distribution of all the excited primary fragments (strongly peaked at the center-of-mass angle slightly larger than $90^{\circ}$ ) there is the rapidly decreasing tail at small angles, see Fig. 8(b). Time distribution for the most damped events $\left(\mathrm{E}_{\text {loss }}>150 \mathrm{MeV}\right)$, in which a large mass transfer occurs and primary fragments scatter in forward angles $\left(\theta_{\text {c.m. }}<70^{\circ}\right)$, is rather narrow and really shifted to longer time delay, see hatched areas in Fig. 7. For the considered case of ${ }^{238} \mathrm{U}+{ }^{248} \mathrm{Cm}$ collision at $800 \mathrm{MeV}$ center-of-mass energy, the detection of the surviving nuclei in the lead region at the laboratory angles of about $25^{\circ}$ and at the low-energy border of their spectrum (around $1000 \mathrm{MeV}$ for $\mathrm{Pb}$ ) could be a real trigger for longest reaction time.

\section{CONCLUSION}

For near-barrier collisions of heavy ions it is very important to perform a combined (unified) analysis of all strongly coupled channels: deep-inelastic scattering, quasi-fission, fusion and regular fission. This ambitious goal has now become possible. A unified set of dynamic Langevin type equations is proposed for the simultaneous description of DI and fusion-fission processes. For the first time, the whole evolution of the heavy nuclear system can be traced starting from the approaching stage and ending in DI, QF, and/or fusionfission channels. Good agreement of our calculations with experimental data gives us hope to obtain rather accurate predictions of the probabilities for superheavy element formation and clarify much better than before the mechanisms of quasi-fission and fusion-fission processes. The determination of such fundamental characteristics of nuclear dynamics as the nuclear viscosity and the nucleon transfer rate is now possible. The production of long-lived neutron-rich SH nuclei in the region of the "island of stability" in collisions of transuranium ions seems to be quite possible due to a large mass rearrangement in the inverse (anti-symmetrized) quasi-fission process caused by the $\mathrm{Z}=82$ and $\mathrm{N}=126$ nuclear shells. A search for spontaneous positron emission from a supercritical electric field of long-living giant quasi-atoms formed in these reactions is also quite promising.

\section{REFERENCES}

1. M. Schädel et al., Phys. Rev. Lett. 41 (1978) 469.

2. M. Schädel et al., Phys. Rev. Lett. 48 (1982) 852.

3. V. Zagrebaev and W. Greiner, J. Phys. G 31 (2005) 825.

4. J. Reinhardt, U. Müller and W. Greiner, Z. Phys. A 303 (1981) 173.

5. J. Maruhn and W. Greiner, Z. Phys. 251 (1972) 431.

6. V.I. Zagrebaev, Phys. Rev. C 64 (2001) 034606.

7. V.I. Zagrebaev et al., Phys. Rev. C 65 (2002) 014607.

8. W.W. Wilcke et al., Phys. Rev. C 22 (1980) 128.

9. V. Zagrebaev and W. Greiner, to be published in J. Phys. G.

10. P. Möller et al., At. Data Nucl. Data Tables 59 (1995) 185.

11. T. Bürvenich et al., Phys. Rev. C 69 (2004) 014307.

12. V.I. Zagrebaev et al., Phys. Rev. C 73 (2006) 031602(R). 